\title{
Effects of anthocyanin-rich grain diet on growth and metastasis of Lewis lung carcinoma in mice
}

Michael V. Tenditnik

Scientific Research Institute of

Physiology and Basic Medicine

Novosibirsk, Russia

m.v.tenditnik@physiol.ru

Nelly A. Popova

Institute of Cytology and Genetics SB RAS

Novosibirsk, Russia

nelly@bionet.nsc.ru
Maria A. Tikhonova

Scientific Research Institute of

Physiology and Basic Medicine

Novosibirsk, Russia

tikhonovama@physiol.ru

Tamara G. Amstislavskaya

Scientific Research Institute of Physiology and Basic Medicine

Novosibirsk, Russia

Amstislavskaya@yandex.ru
Ekaterina A. Litvinova

Scientific Research Institute of Physiology and Basic Medicine

Novosibirsk, Russia

litvinovaea@physiol.ru

Elena K. Khlestkina

N.I. Vavilov All-Russian Research Institute of Plant Genetic Resources

St. Petersburg, Russia

Institute of Cytology and Genetics SB RAS

Novosibirsk, Russia

khlest@bionet.nsc.ru

\begin{abstract}
Currently functional nutrition has developed intensively. Functional foods are a valuable addition to existing dietary therapy. Such products are enriched with biologically active substances. Anthocyanins attract particular attention due to multiple beneficial properties including antitumor activity. Here we used two wheat near-isogenic lines created at the Institute of Cytology and Genetics SB RAS [1] that have almost similar genomes with the exception of a small part of chromosome $2 \mathrm{~A}$, which contains the $\mathrm{Pp3} / \mathrm{TaMyc1}$ gene regulating anthocyanin biosynthesis, to assess the effect of an anthocyanin-rich grain diet on the development and metastasis of the Lewis lung carcinoma (LLC), as well as the accompanying

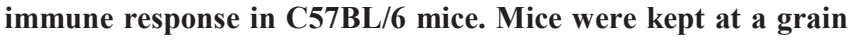
or standard diet for four month prior tumor transplantation. A decrease in the number of metastases in the lungs and the size of the tumor in the groups at a grain diet, regardless of the content of anthocyanins, was revealed. However, the highest percentage of animals without metastases was observed at an anthocyaninrich grain diet. The LLC transplantation caused a significant increase in plasma levels of pro-inflammatory cytokines (IL-6
\end{abstract}

and LIF) in mice at a standard diet, but not in animals at grain diets. Thus, the anthocyanin-rich diet helps to reduce the severity of the tumor process. The antitumor effects of grain diets are probably mediated by modulation of signaling pathways associated with IL-6 cytokines.

Keywords - functional food, wheat grain, anthocyanins, tumor, metastasis, mice, immune, cytokine.

\section{ACKNOWLEDGMENT}

The study was supported by Russian Science Foundation (grant No. 16-14-00086).

\section{REFERENCES}

[1] E. I. Gordeeva, O. Y. Shoeva, and E. K.Khlestkina, "Marker-assisted development of bread wheat near-isogenic lines carrying various combinations of purple pericarp $(P p)$ alleles," Euphytica, vol. 203, pp. 469-476, 2015. 